\title{
Cryogenic STM in 3D vector magnetic fields realized through a rotatable insert
}

\author{
C. Trainer, ${ }^{1}$ C. M. Yim, ${ }^{1}$ M. McLaren, ${ }^{1}$ and P. Wahl ${ }^{1,}$ a) \\ SUPA, School of Physics and Astronomy, University of St Andrews, North Haugh, St Andrews, Fife KY16 9SS, \\ $U K$
}

(Dated: 7 September 2017)

Spin-polarized scanning tunneling microscopy (SP-STM) performed in vector magnetic fields promises atomic scale imaging of magnetic structure, providing complete information on the local spin texture of a sample in three dimensions. Here, we have designed and constructed a turntable system for a low temperature STM which in combination with a $2 \mathrm{D}$ vector magnet provides magnetic fields of up to $5 \mathrm{~T}$ in any direction relative to the tip-sample geometry. This enables STM imaging and spectroscopy to be performed at the same atomic-scale location and field-of-view on the sample, and most importantly, without experiencing any change on the tip apex before and after field switching. Combined with a ferromagnetic tip this enables us to study the magnetization of complex magnetic orders in all three spatial directions.

PACS numbers: 07.79.Cz, 75.70.Tj, 68.37.-d

Keywords: spin-polarized scanning tunneling microscopy, magnetism, vector magnetic fields

\section{INTRODUCTION}

Spin polarized scanning tunneling microscopy (SP$\mathrm{STM})$ is a powerful experimental technique that is capable of resolving the magnetic structure of a sample down to the atomic scale. Since the early days of SP-STM when the ferromagnetic (FM) terraces on the $\operatorname{Cr}(001)$ surface were first observed, 1 the technique has evolved, developing the ability to resolve ever more complex and exotic magnetic phenomena that occur on atomic length scales which are invisible to conventional bulk probing techniques such as neutron scattering. To name a few, SP-STM has been employed to probe the magnetization of nanoscale cobalt islands, ${ }^{2}$ to measure the magnetic hysteresis in FM nanostructures, $\stackrel{3,4}{3}$ and to study spin-dependent quasi-particle interference. ${ }^{5}$ Recently, SP-STM has also been applied to study strongly correlated electron materials, ${ }^{6 / 7}$ providing further insights into the exotic phases found in these materials. ${ }^{8}$

SP-STM operating with a vector magnet has been extensively used to map the local surface spin textures in different systems, including the chiral magnetic order that develops in a Mn monolayer formed on $\mathrm{W}(110), 9$, the "stripe-like" antiferromagnetic (AFM) order present in iron-based superconducting materials, ${ }^{7}$, and the Neél-type AFM order in FeSe thin-film grown on the $\mathrm{SrTiO}_{3}(111)$ surface $\frac{10}{10}$ In those works, in order to map the local surface spin texture of the sample, the magnetization of the spin-polarized STM tip was manipulated by an applied field. If the magnetic structure of the sample is unaltered by the applied field, then the STM tip, which has been polarized along the direction of the applied field, is able to image the component of the surface spin texture along the field direction. Provided

\footnotetext{
a)Electronic mail: wahl@st-andrews.ac.uk
}

that magnetic fields can be applied along three orthogonal directions with respect to the tip-sample geometry, mapping the surface magnetic structure of a sample in three directions is possible.

To be able to perform SP-STM measurements one must first have a spin-polarized tip. There are different ways of preparing spin-polarized STM tips ${ }^{11}$ These include coating a non-magnetic tip (usually made of $\mathrm{W}$ or PtIr) with a magnetic material, $\sqrt{7912]}$ or fabricating a tip out of a magnetic material ${ }^{13}$ The advantage of preparing spin-polarized tips by coating is that, depending on the type of the magnetic structure under study, one can form a spin-polarized tip of different magnetic properties by choosing the appropriate coating material. ${ }^{7912}$ For example by using Fe, a FM tip which can be polarized by an applied field is obtained ${ }^{79}$. Alternatively by using $\mathrm{Cr}$ an AFM tip which is capable of imaging magnetic features that are not robust against applied field is achieved ${ }^{12}$. It is also possible to form a spin-polarized tip out of a bulk magnetic material. ${ }^{[13}$ This has the advantage of not requiring a UHV environment but the magnetization of such a tip may not be trivial to manipulate, which in turn reduces its versatility for use.

Another method of preparing spin-polarized tips insitu is to pick up material from a magnetic sample, achieved by using voltage pulses or high tunneling current scanning of the tip across the sample ${ }^{6[14 \mid 15}$ Our method of preparing spin polarized tips is to collect either excess Fe (FM tip) or FeTe clusters (AFM tip) from the surface of an FeTe reference sample ${ }^{14}$. This method is capable of producing soft magnetic tips that can be polarized by a small magnetic field $(\sim 0.5 \mathrm{~T})$, it can reproducibly produce spin-polarized tips of different (either FM or AFM) characters, and after tip preparation, characterization of the tip can be performed on the same sample.

The working principle of SP-STM is based on the dependence of the tunneling current on the relative orientation of the magnetization of the two spin-polarized 
electrodes: i.e. maximal (minimal) tunneling current is achieved when the magnetization of the two electrodes are aligned parallel (anti-parallel) to each other ${ }^{11}$ As a result, by imaging the same position on a magnetic sample twice, first with the tip polarized by a magnetic field applied along one direction and then with it re-polarized by a field applied along the opposite direction, the component of the surface magnetic structure parallel to the field direction is then obtained by taking the difference of the two images. An elegant way to achieve this has been employed previously by periodically changing the magnetization of the tip. In this method, topographic and spin-dependent parts of the tunneling current are readily separated using a lock-in technique, enabling simultaneous acquisition of topographic and magnetic-structure images. $\frac{16117}{\text {. }}$.

The capability to apply magnetic field in any direction relative to the sample in the STM allows for mapping of the surface spin texture in three dimensions 10 . 3D vector magnets have in recent years become commercially available, and the construction of low temperature STMs with 3D vector magnets $\sqrt{18 \mid 19}$ has been reported. $3 \mathrm{D}$ vector magnets are however usually rather bulky, do not allow for arbitrary field vectors in excess of $1 \mathrm{~T}$ and often result in rather high helium boil-off due to the need for six magnet leads. Here, we circumvent these limitations by the construction of a turntable system, which, combined with a $2 \mathrm{D}$ vector magnet, allows for the application of arbitrary field vectors of up to $5 \mathrm{~T}$ relative to the sample. In this way we demonstrate imaging of the same atomic-scale location of the sample with the same tip for different field orientations, evidencing the viability of this approach.

\section{STM INSERT}

The cryogenic insert and STM head of our vector magnet STM are constructed based on the design of White et al. ${ }^{20}$. As shown in Figure 1, the STM head is made of sapphire which, owing to its high stiffness at comparatively low mass, yields a high resonance frequency, leading to excellent mechanical vibration isolation of the tunneling junction, as well as good thermal conductivity. The non-magnetic nature of sapphire also makes it ideal as a building material for the STM head to minimize the influence of the magnetic field.

Inside the assembly, an STM tip, usually made of W or $\mathrm{Pt} / \mathrm{Ir}$ wire, is placed on top of a piezoelectric scanner tube housed in a sapphire prism. Coarse motion of the STM tip along the vertical direction is realized using a Panstyle piezo motor ${ }^{21}$. Fine motion of the STM tip along three orthogonal directions is then achieved by careful application of voltages to each of five electrodes of the scanner tube. The STM assembly is then placed in a vacuum-sealed cryogenic insert housed in a liquid helium cryostat equipped with a $2 \mathrm{D}$ vectormagnet. With the use of a $1 \mathrm{~K}$ pot cooling system, the STM head can be cooled down to a temperature of $\sim 1.6 \mathrm{~K}$. Conventionally, the cryogenic insert is bolted to the cryostat and sealed by a viton gasket.

Different from the sample plate of a typical STM head constructed as outlined by White et al ${ }^{20}$, the sample plate provides four separate electrical contacts to the sample (see Fig. 22). Apart from standard STM operation, this also allows for simultaneous transport measurement as well as providing flexibility for more complex devices to be measured using the STM.

Pristine, atomically flat surfaces are prepared by in-situ sample cleaving at $\sim 20 \mathrm{~K}^{200}$, achieved by using a smart sample cleaving mechanism located at the middle part of the cryogenic insert. Sample transfer is performed using a differentially-pumped manipulator.

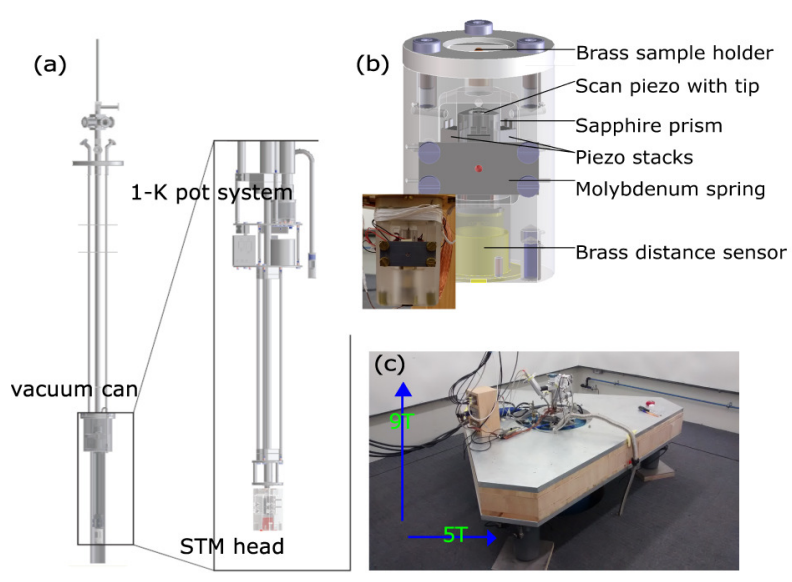

FIG. 1. (a) Computer rendering of the cryogenic insert. Inset: zoom-in drawing of the internal part of the insert that houses all the STM electronics and the $1 \mathrm{~K}$ pot cooling mechanism through which a base temperature of $\sim 1.6 \mathrm{~K}$ is achieved. (b) Computer rendering and photograph of the STM measurement head. Its body is made of sapphire, which provides high thermal conductivity and a high resonant frequency, the latter of which prevents the transmission of low frequency noise to the tunnel junction. (c) Photograph of the STM setup with a pictorial representation of the maximal fields that can be applied along the in-plane and out-of-plane directions by the $2 \mathrm{D}$ vectormagnet.

\section{TURNTABLE}

The 2D vectormagnet of our instrument is a two coil Maxes MX-2 Magnet system 22] This allows for the application of magnetic fields of \pm 9 and $\pm 5 \mathrm{~T}$ along the vertical $\left(B_{\perp}\right)$ and horizontal $\left(B_{\|}\right)$directions respectively (see Fig. 3a). As demonstrated in the schematics in Fig. 3a, by applying fields in both directions at the same time, application of a magnetic field of $5 \mathrm{~T}$ pointing to any direction on a vertical circular orbit can be achieved. 

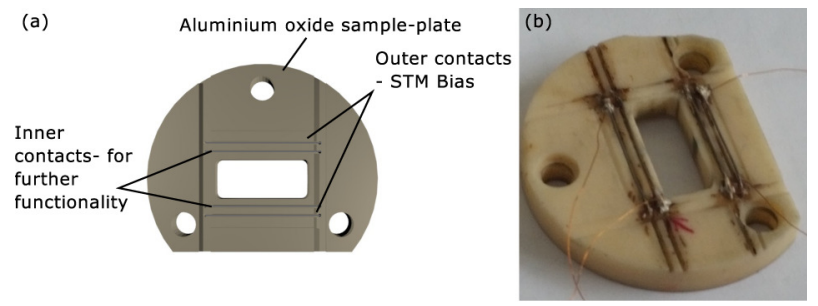

FIG. 2. (a) Computer rendering of the Aluminum oxide sample plate of the STM head. The sample plate provides four independent electrical contacts to the sample which allows for a variety of different measurements to be carried out on the sample. Furthermore it also provides scope for the insertion of future devices. (b) Photograph depicting the modified sample plate.

Coupling this with the rotation of the cryogenic insert relative to this orbit (see Fig 3p) it is possible to apply a field of up to $5 \mathrm{~T}$ in any given direction relative to the sample under study.

(a)

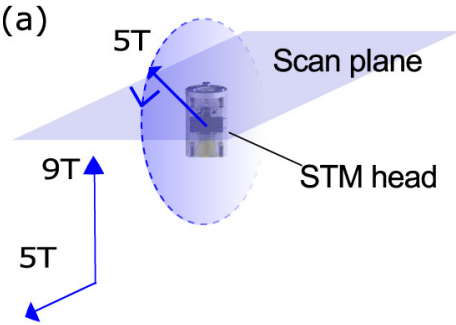

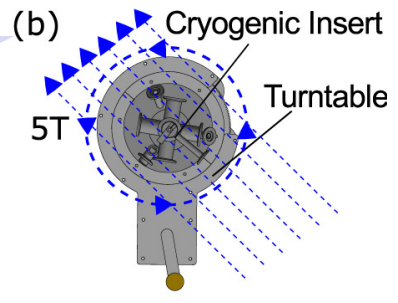

FIG. 3. (a) Schematic showing the applicable fields relative to the STM head that can be applied by the unmodified vectormagnet. (b) Schematic showing how the STM insert is rotated with respect to the fixed in-plane field.

To realize the rotation of the insert, without loosing the atomic-scale position and maintaining the same tip apex, we have constructed a turntable device to rotate the insert while protecting it from any sudden mechanical shocks as would almost certainly occur if the insert was lifted for rotation as well as ensure proper sealing of the insert to the cryostat to prevent helium loss. As shown in Fig. 4 the turntable device comprises three main parts: the top and bottom plates, and the main gear. The main gear, comprising of a pair of half rings with teeth on the outer peripheries and rotation scale markers at the top, is slotted onto the top flange of the cryogenic insert. Instead of having the cryogenic insert bolted directly to the magnet dewar it is inserted into the LHe cryostat through a sliding seal and made Helium tight by a viton O-ring between the insert and the cryostat. The insert is then held in position by sandwiching it between the top and bottom plates of the turntable that are bolted to each other. The bottom plate is welded to the top of the magnet dewar beforehand. Rotation of the cryogenic insert is then achieved by rotating the main gear through a set a small gears using a crank handle positioned on the top plate. This provides a rotation resolution of $0.5^{\circ}$.

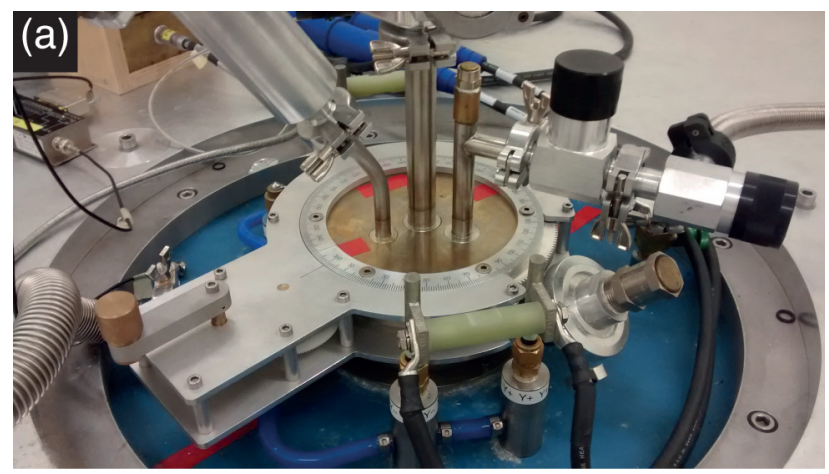

(b) Top plate

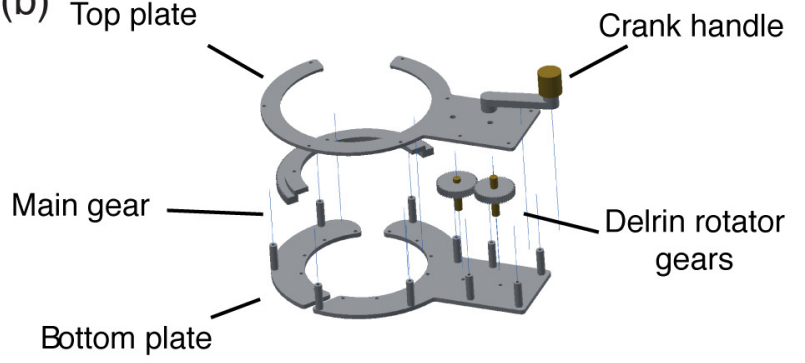

FIG. 4. (a) Assembled turntable mechanism. (b) Exploded computer rendering showing the relationship of the different parts of the turntable.

With this setup, the cryogenic insert, and hence the STM assembly, can be rotated freely along the azimuthal direction relative to the direction along which the inplane magnetic field is applied, while everything remains coaxial with each other. As such, by combining the use of the turntable system with the application of the inplane and out-of-plane fields of the $2 \mathrm{D}$ vector-magnet, the application of a magnetic field that points along any direction with respect to the sample in the STM assembly is achieved.

The operating procedure of the turntable setup is as follows: first, the tip is taken out of tunneling and retracted by about $150 \mathrm{~nm}$; then, the STM insert is rotated by a desired angle with the use of the turntable; last the tip is brought back to tunneling conditions and measurement is resumed. Despite mechanically moving the STM head in the field, for changes of the angle on the order of tens of degrees the scanned area of our STM experiences a lateral shift of only a few $\mathrm{nm}$ during this process, requiring only minor adjustment of the scan area to acquire images in the same location. This is likely due to only very small Eddy-current heating, as the field change is rather minor, as well as a consequence of the STM head being entirely made out of non-magnetic materials.20 

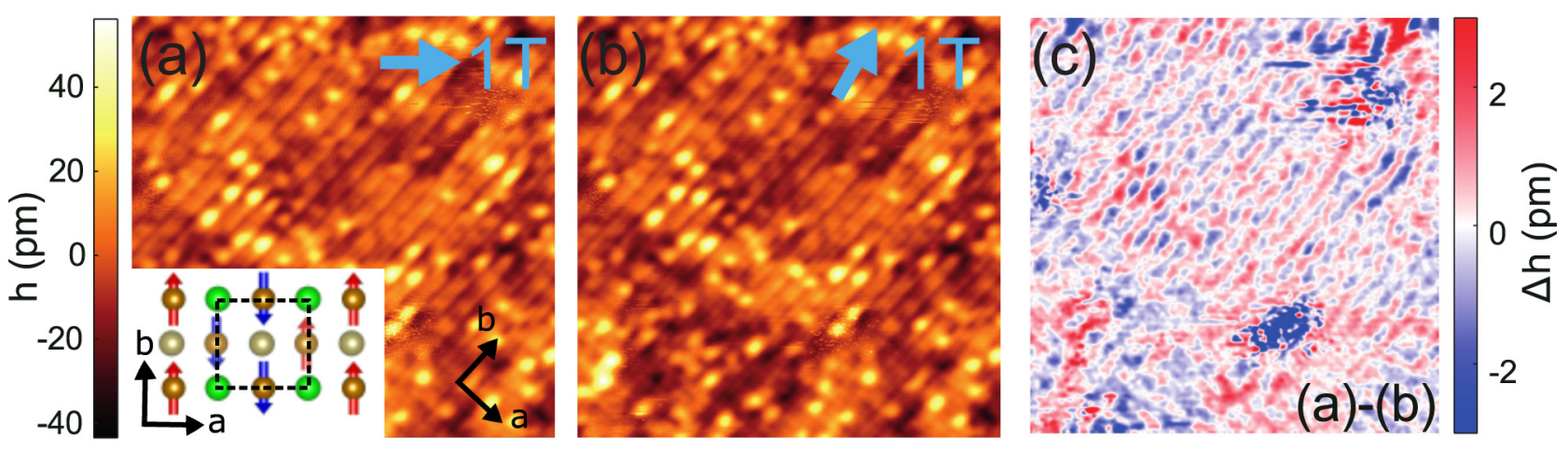

FIG. 5. (a) $15 \times 15 \mathrm{~nm}^{2} \mathrm{STM}$ images of the $\mathrm{Fe}_{1.06}$ Te surface taken at an in-plane field of $+1 \mathrm{~T}$. Inset: schematic of the unit cell of the surface FeTe layer, where green and gold spheres represent the top and bottom Te atoms and brown spheres the Fe atoms. Red and blue arrows represent the spins of the iron atoms pointing up or down, respectively, in the bi-collinear AFM order. (b), as (a), recorded after the STM insert was rotated by $60^{\circ}$ from its original azimuth. Both images were taken using a FM spin-polarized tip, at $1.6 \mathrm{~K}(U=0.1 \mathrm{~V}, I=50 \mathrm{pA})$. (c) Difference image formed by subtracting the image in (a) by that in (b), showing the difference in magnetic contrast between the two field directions.

\section{PERFORMANCE}

To demonstrate operation of this turntable system and that the design criteria are met, we show here STM images obtained on the surface of iron tellurium $\left(\mathrm{Fe}_{1+x} \mathrm{Te}\right.$, where $\mathrm{x}=0.06)$, the non-superconducting parent compound of iron chalcogenide superconductors. As shown in the ball model in the inset of Fig. $5 \mathrm{a}, \mathrm{Fe}_{1.06} \mathrm{Te}$ exhibits a bi-collinear stripe magnetic order with wave vector $\mathbf{q}=(1 / 2,0,1 / 2)$. Imaging of the magnetic order of $\mathrm{Fe}_{1+x} \mathrm{Te}$ in STM was previously reported by Enayat et $a l$. ${ }^{6}$ When the sample is imaged with a nonmagnetic tip, the Te atoms at the topmost layer dominate the image contrast, leading to a square atomic lattice showing up in the image. However, when a magnetic tip is used, the contrast of STM images contains contributions from the magnetic order of the sample. The magnetic order manifests itself as stripes running along the crystal $b$-axis in STM images. The intensity of these stripes is dependent on the relative spin orientation of the tip and the sample. By rotating the applied magnetic field, achieved here by rotating the insert, we thereby change the spin orientation of the tip with respect to the sample and hence the intensity with which the magnetic order is imaged changes.

Figures $5 \mathrm{a}-\mathrm{b}$ show two STM images of the $\mathrm{Fe}_{1+x} \mathrm{Te}$ surface obtained using our turntable setup with a magnetic tip apex. Methods for sample growth and preparation were described elsewhere $e^{6}$. The magnetic tip apex was prepared by picking up some excess $\mathrm{Fe}$ atoms from the surface of $\mathrm{Fe}_{1+x}$ Te. Both STM images were recorded in the presence of $\mathrm{a}+1 \mathrm{~T}$ in-plane field. However, one image (Fig. 5 a) was collected at a turntable angle at which the STM assembly is aligned parallel to the field direction, while the other (Fig. 5b) was obtained at a different turntable angle at which the STM assembly is $60^{\circ}$ away from the field direction. Both STM images exhibit stripes, confirming the magnetic nature of the tip. However, after rotation of the turntable, the spin-polarized component of the tunneling current has become weaker (as evident from the difference image in Fig 58). Such change arises from the change in the angle between the magnetization of the spin-polarized tip and the AFM order of the $\mathrm{Fe}_{1.06}$ Te sample. Most importantly, before and after rotation, the STM tip does not experience any change, and the same atomic scale area on the surface can be found with only minimal drift of the tip position on the order of $\sim 1 \mathrm{~nm}$ that develops as a result of the rotation.

\section{SUMMARY}

In conclusion, we have designed and constructed a turntable system which, when employed together with a low temperature STM installed in a $2 \mathrm{D}$ vectormagnet, allows for full rotation of the STM with respect to the applied in-plane field whilst preserving the STM tip and scanned location on the sample. Altogether these enable the full mapping of surface magnetic structure in three dimensions. Our setup thus provides a way to increase the achievable vector magnetic fields for low temperature scanning tunneling microscopy.

\section{ACKNOWLEDGEMENTS}

We acknowledge funding from EPSRC (EP/L505079/1 and $\mathrm{EP} / \mathrm{I} 031014 / 1)$. $\mathrm{Fe}_{1.06} \mathrm{Te}$ samples have been obtained from V. Tsurkan and A. Loidl, University of Augsburg.

\footnotetext{
${ }^{1}$ R. Wiesendanger, H.-J. Güntherodt, G. Güntherodt, R. J. Gambino, and R. Ruf, Phys. Rev. Lett. 65, 247 (1990).

${ }^{2}$ O. Pietzsch, A. Kubetzka, M. Bode, and R. Wiesendanger, Phys. Rev. Lett. 92, 057202 (2004).
} 
${ }^{3}$ O. Pietzsch, A. Kubetzka, M. Bode, and R. Wiesendanger, Science 292, 2053 (2001).

${ }^{4}$ J. Prokop, A. Kukunin, and H. J. Elmers, Phys. Rev. Lett. 95, 187202 (2005).

${ }^{5}$ H. Oka, P. A. Ignatiev, S. Wedekind, G. Rodary, L. Niebergall, V. S. Stepanyuk, D. Sander, and J. Kirschner, Science 327, 843 (2010).

${ }^{6}$ M. Enayat, Z. Sun, U. R. Singh, R. Aluru, S. Schmaus, A. Yaresko, Y. Liu, C. Lin, V. Tsurkan, A. Loidl, J. Deisenhofer, and P. Wahl, Science 345, 653 (2014).

${ }^{7}$ T. Hänke, U. R. Singh, L. Cornils, S. Manna, A. Kamlapure, M. Bremholm, E. M. J. Hedegaard, B. B. Iversen, P. Hofmann, J. $\mathrm{Hu}$, Z. Mao, J. Wiebe, and R. Wiesendanger, Nat. Commun. 8, 13939 (2017).

${ }^{8}$ E. Dagotto, Science 309, 257 (2005).

${ }^{9}$ M. Bode, M. Heide, K. von Bergmann, P. Ferriani, S. Heinze, G. Bihlmayer, A. Kubetzka, O. Pietzsch, S. Blügel, and R. Wiesendanger, Nature 447, 190 (2007).

${ }^{10}$ K. F. Zhang, X. Zhang, F. Yang, Y. R. Song, X. Chen, and C. Liu, D. Qian, W. Luo, C. L. Gao, and J-F. Jia, Appl. Phys. Lett. 108061601 (2016).

${ }^{11}$ R. Wiesendanger, Rev. Mod. Phys. 81, 1495 (2009).

${ }^{12}$ A. Kubetzka, M. Bode, O. Pietzsch, and R. Wiesendanger, Phys. Rev. Lett. 88, 057201 (2002).
${ }^{13}$ R. Wiesendanger, I. V. Shvets, D. Bürgler, G. Tarrach, H. J. Güntherodt, J. M. D. Coey, and S. Gräser, Science 255, 583 (1992).

${ }^{14}$ U. R. Singh, R. Aluru, Y. Liu, C. Lin, and P. Wahl, Phys. Rev. B 91, 161111 (2015).

${ }^{15}$ T. K. Yamada, M. M. J. Bischoff, T. Mizoguchi, and H. van Kempen, Appl. Phys. Lett. 82, 1437 (2003).

${ }^{16}$ H. F. Ding, W. Wulfhekel, and J. Kirschner, EPL 57, 100 (2002).

${ }^{17}$ W. Wulfhekel, and J. Kirschner, Appl. Phys. Lett. 75, 1944 (1999).

${ }^{18}$ J. A. Galvis, E. Herrera, I. Guillamón, J. Azpeitia, R. F. Luccas, C. Munuera, M. Cuenca, J. A. Higuera, N. Díaz, M. Pazos, M. García-Hernandez, A. Buendía, S. Vieira and H. Suderow, Rev. Sci. Instrum. 86, 013706 (2015).

${ }^{19}$ S. Meckler, M. Gyamfi, O. Pietzsch, and R. Wiesendanger, Rev. Sci. Instrum. 80, 023708 (2009).

${ }^{20}$ S. C. White, U. R. Singh, P. Wahl, Rev. Sci. Instrum. 82, 113708 (2011).

${ }^{21}$ S.-H. Pan, Patent, WO 93/19494 (1993).

${ }^{22}$ American Magnetics, Inc., Oak Ridge, TN. 